\section{Defensive medicine in the emergency department. The clinicians' perspective}

\author{
Gianfranco Cervellin, ${ }^{1}$ Mario Cavazza ${ }^{2}$ \\ 'Emergency Department, Parma \\ University Hospital, Parma; ${ }^{2}$ Emergency \\ Department, S. Orsola University \\ Hospital, Bologna, Italy
}

\section{Abstract}

The overuse of medical services is regarded as a growing problem in Western countries, accounting for up to $30 \%$ of all delivered care, and carrying a higher risk of morbidity and mortality. One of the leading drivers toward medical overuse is the so-called defensive medicine, which is commonly defined as ordering of tests, procedures, and visits, or, at variance, avoidance of high-risk patients or procedures, aimed to reduce exposure to malpractice liability. Defensive medicine may increase the amount of care provided to the patients (i.e., additional tests or therapies), change care or setting of care (i.e., patients referred to another specialist or another healthcare facility), or impair the optimal care (i.e., refusing risky patients). Some studies seem to confirm a large utilization of defensive medicine in the emergency departments. This article tries to analyze some key points capable to pave the way to a consistent reduction of defensive medicine, thus defining a hierarchical list of priorities, keeping the patient's health always at the center of the matter.

\section{Introduction}

The sunset of the medical paternalism, strictly linked with the self-referentiality of all the medical practice, has apparently paved the way for the emerging problem of the so-called medical overuse and defensive medicine. The patients are indeed increasingly informed about medical issues, and less likely to accept at face value what is being recommended, thus creating new problems for the doctors, who are not conventionally used to have their professional judgment and integrity challenged. It has been suggested that As a group, physicians are acutely sensitive to any suggestions that they have failed to meet the standard of care or are not 'good' doctors... This accusation of failure represents a personal assault. ${ }^{1}$ The media play a pivotal role in this vicious circle, giving increasing strength to sensationalism and underlining with special emphasis the news about malpractice (although seldom confirmed), or else disseminating questionable information about new and phenomenal treatments, which obviously rises patients' expectations. $^{2}$

The overuse of medical services, defined as care in the absence of a clear medical basis for use or when the benefit of therapy does not outweigh risks, ${ }^{3}$ is regarded as a growing problem in Western countries, accounting for up to $30 \%$ of all delivered care. ${ }^{4-6}$ Incidentally, it is has also been acknowledged that medical overuse carries a higher risk of morbidity and mortality. ${ }^{7,8}$ The prescription of magnetic resonance for simple low back pain is a paradigmatic medical action, which may be associated with useless and potentially harmful surgical management.

One of the best recognized drivers toward medical overuse is the so-called defensive medicine,${ }^{9}$ which is commonly defined as: i) ordering of tests, procedures, and visits, or, ii) avoidance of high-risk patients or procedures, primarily to reduce exposure to malpractice liability. ${ }^{10}$ The former is commonly known as assurance behavior, or positive defensive medicine, and is characterized by supplying additional tests and/or treatments of marginal or no medical value with the main target of reducing adverse outcomes, discouraging patients from suing the doctors, or convincing the lawyers that the standard of care was met. The latter, conversely, is widely known as avoidance behavior or negative defensive medicine, and reflects the physicians' efforts to refuse to treat particular (i.e., risky) patients, or to refer them to another physician or health facility to escape the challenge. As such, defensive medicine may have different consequences: it may increase the amount of care provided to the patients (i.e., additional tests or therapies), change care or setting of care (i.e., patients referred to another specialist or another healthcare facility), or, finally, impair the optimal care (i.e., refusing risky patients). Defensive medicine has been studied in different specialties settings, with patchy results. ${ }^{11}$

Although widely condemned, defensive medicine is inextricably interwoven into daily medical practice, and it can often be unconscious and involuntary. ${ }^{12,13}$ Many factors may contribute to this attitude, including the lack of information about specific tests and/or treatments, lack of communication between different specialists, lack of knowledge of guidelines and so on. The physicians may hence practice defensive medicine without guilt, being their action neither unacknowledged, nor considered unethical. Should then we consider medical ignorance as an innocent and genuine behavior? Who should establish the boundaries between medical ignorance and adequate or suitable medical culture?
Correspondence: Gianfranco Cervellin, Emergency Department, Parma University Hospital, via Gramsci 14, 43126 Parma, Italy. Tel: +39.0521 .703800 .

E-mail: gcervellin@ao.pr.it; gianfranco.cervellin@gmail.com

Key words: Defensive medicine; Emergency medicine; Emergency department; Diagnostic testing; Overtreatment.

Received for publication: 30 October 2015. Accepted for publication: 31 December 2015

This work is licensed under a Creative Commons Attribution 4.0 License (by-nc 4.0).

(C) Copyright G. Cervellin and M. Cavazza, 2016 Licensee PAGEPress, Italy

Emergency Care Journal 2016; 12:5615

doi:10.4081/ecj.2016.5615

According to an economic perspective, defensive medicine is widely considered as a major source of wasteful medical spending in the Western World. Solely in the United States, for example, a widely cited report estimates that $\$ 210$ billions are spent annually on needless care motivated by fear of malpractice liti-

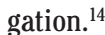

\section{Opinion Report}

\section{Defensive medicine in emergency settings}

As regards the practice of defensive medicine in the Emergency Department (ED), the largest study ever published to date is that published by Studdert and coworkers in 2005..$^{15}$ The Authors observed that a group of emergency physicians (EPs) reported that they often practice defensive medicine, both with assurance and with avoidance behaviors. Notably, the proportion of doctors ordering more diagnostic tests than were medically indicated was significantly higher for EPs than for other specialists (i.e., general surgeons, orthopedic surgeons, neurosurgeons, obstetrician/gynecologists). As specifically regards assurance behavior, the EPs indicated that more tests than those medically indicated were ordered in $70 \%$ of cases, more medications (e.g., antibiotics) than those medically indicated were prescribed in $30 \%$ of cases, patients were referred to other specialists in unnecessary circumstances in $52 \%$ of cases, and invasive procedures (e.g., biopsies) to confirm diagnoses were suggested in $19 \%$ of cases. As regards avoidance behavior, $21 \%$ of EPs indicated that certain procedures or interventions were often avoided, whereas $13 \%$ of them also 
avoided providing care to high-risk patients. It is noteworthy, however, that the choice of patients (e.g., the possibility to decline care to some of them) is not an option in most countries, since emergency medicine is inherently driven by urgent needs and not by other considerations.

The specific practices of defensive medicine mostly involved frequent orders of computed tomography (CT), magnetic resonance imaging (MRI), or X-ray without clear medical indications in $63 \%$ of cases, admission of patients without clear indication in $14 \%$ of cases, performance of cardiac workup in $12 \%$ of cases, and patients referral to another physician in $5 \%$ of cases. Once more, this last issue needs additional comments, since referring the patient to another physician may be simply interpreted as the action of discharging responsibilities, but also as lengthening the work-up so leading to the change of work-shift.

In the same study, objective measures of physicians' liability experience and exposure were not associated with individual propensity to practice defensively. ${ }^{15}$ This finding is quite surprising, suggesting that personal anxiety may overshadow actual experience, as proposed by Glassmanand colleagues. by the notion that the signal to practice defensively may have been broadcast so widely that individual experience is overshadowed by collective anxiety. ${ }^{16}$ Moreover, EPs feel compelled to practice maximally intensive medicine according to what they believe their peers are doing since malpractice is usually defined as a deviation from the standard of customary practice.

EPs typically practice in an informationpoor, high-risk, technology-rich environment, a setting that may predispose itself to defensive practice and magnify expenditures. The EP's decision to admit a patient to the hospital is associated with inpatient costs that may be 10 times higher than the ED visit itself. ${ }^{17}$ It is now almost clear that EPs tend to practice defensive medicine in several aspects of their daily work. To put it simple, some of us know it, acknowledge it, and accept it, whereas others do not.

The ED, as previously mentioned, is a clinical setting characterized by a high technology environment, and technology itself plays a key role in defensive medicine, and in malpractice liability in general. ${ }^{18}$ The EPs often usee technology to pacify demanding patients, bolster their own self-confidence, or create a trail of evidence that they had confirmed or excluded particular disease entities. The defensive use of technology, however, is self-reinforcing. The larger is the number of diagnostic procedures with low predictive values or aggressive treatments for low-risk conditions, the more likely is that such practices become the legal standard of care. This represents the classical $d o g$ chasing its own tail figure.
Trauma evaluation is one of the fields of Emergency Medicine in which defensive medicine is most practiced. Some evidence exist, however, that the enormous increase in the use of CT does not reflect a similar increase in efficient diagnosis, ${ }^{19}$ whilst it exposes the patients to potentially unnecessary amounts of harmful radiation. However, out of the 416 CT studies ordered for defensive purposes, 51 (12\%) had significant findings, leading in change of care in 9 (2.2\%). As such, 9 out of 416 patients should be seen as potential lawsuits. ${ }^{19}$

An additional field of particular interest in emergency (defensive) medicine is the evaluation of chest pain patients. Due to the fear of discharge patients at high risk even after exclusion of a myocardial infarction (MI), an extensive work-up is quite often performed, including provocative tests during the observation period. Nevertheless, a large study including 421,774 patients failed to show any benefit from such an aggressive strategy in those patients, concluding that this population does not appear to be affected by the initial testing strategy. Deferral of early noninvasive testing appears thus to be reasonable. ${ }^{20}$

\section{Setting an agenda for reducing defensive (emergency) medicine?}

Since defensive medicine violates the Hippocratic oath and represents a serious challenge to the physician-patient relationship, an agenda for reducing its use is urgently needed.

The Office of Technology Assessmenty definition of defensive medicine ${ }^{21}$ (see previous part of this article) means that defensive medicine should be regarded a negative shift from the ideal medical practice. Now, the question is: who defines ideal medical practice, or standard of care? Physicians, patients, hospital managers, or lawyers? Rather reasonably, we think that standard of care can be seen as a mix of two components, i.e., i) what is medically justified in defined clinical situations, and, ii) what is legally required, that reflects community/customers' expectations. ${ }^{22}$

There is a widespread perception that courts tend to rely more on data provided by instrumental or laboratory tests than on claims of experience or medical judgment. ${ }^{23}$ It is also commonplace to think that defensive medicine is mainly driven by fear of lawsuits. There are few data, however, to confirm this perception, and the limited weight of evidence suggests that the effect is limited. ${ }^{24} \mathrm{~A}$ recent and large study evaluating the effects of new laws on malpractice in three US states (i.e., Texas, Georgia, and South Carolina), failed to demonstrate any significant effect on defensive medicine practice. More specifically, the study showed that a new legislation substantially changing the malpractice standard for EPs had little, if any, effect on the intensity of practice, as measured by imaging rates, average charges, or hospital admission rates. ${ }^{25}$ These findings seems to mine the concept, otherwise widely accepted, that the fear of being involved in medico-legal litigation is the main driver of defensive medicine.

Evidence seems to suggest that the clinical history typically accounts for $75 \%$ or more of the diagnostic yield when evaluating common symptoms, the physical examination 10 to $15 \%$, and testing generally less than $10 \% .{ }^{26}$ As such, clinical Gestalt and clinical judgement still retain a pivotal role in each clinical decision, albeit corroborate by Bayesian principles, with different weight in different clinical situations. ${ }^{27}$ Venous thromboembolism ${ }^{28}$ and acute myocardial infarction ${ }^{29}$ are two valuable examples of severe disorders, highly prevalent in the setting of emergency medicine, whose diagnosis and management has taken great advantage from the introduction of diagnostic tests, algorithms and guidelines, which should work side-by-side with clinical Gestalt and clinical judgement.

\section{Conclusions}

In order to optimize our practice, thus paving the way to a consistent reduction of defensive medicine, we should define a hierarchical list of priorities, in which the patient's health is, and will always be, at the center. We are physicians before all, and we should not forget that although clinical instinct and experience play a pivotal role in the formulation of initial impressions and management, the precious contribution of evidence-based medicine should not be awkwardly overlooked. The clinical reasoning should hence be regarded as a virtuous circle, whereby the Gestalt leads to the use of available scientific tools, which in turn may reinforce (or even weaken) the initial Gestalt perception. This has been strongly reaffirmed by the US Institute of Medicine, by listing a set of core competencies for health professions education, which include interdisciplinary teams, evidence-based practice, quality improvement and informatics along with patient centered care..$^{30}$ This kind of educational policy should, of course, start from the University, since a large portion of medical students and residents frequently encounter defensive medicine practices and are often being taught to consider malpractice liability during clinical decision making. ${ }^{31}$ Along with revision of current legislation, getting back to basics may be the best way to fight against defensive medicine. 


\section{References}

1. Charles SC. Coping with a medical malpractice suit. Western J Med 2001;174:55-8.

2. Toraldo DM, Vergari U, Toraldo M. Medical malpractice, defensive medicine and role of the "media" in Italy. Multidiscip Respir M 2015;10:12.

3. Morgan DJ, Brownlee S, Leppin Al, et al. Setting a research agenda for medical overuse. Brit Med J 2015;351:4534.

4. Chassin MR. Is health care ready for six sigma quality? Milbank Q 1998;76:565-91.

5. Berwick DM, Hackbarth AD. Eliminating waste in US health care. J Am Med Assoc 2012;307:1513-6.

6. Sirovich BE, Woloshin S, Schwartz LM. Too little? Too much? Primary care physicians' views on US health care: a brief report. Arch Intern Med 2011;171:1582-5.

7. Welch HG, Sharp SM, Gottlieb DJ, et al. Geographic variation in diagnosis frequency and risk of death among medicare beneficiaries. J Am Med Assoc 2011;305: 1113-8.

8. Song Y, Skinner J, Bynum J, et al. Regional variations in diagnostic practices. New Engl J Med 2010;363:45-53.

9. PerryUndem Research/Communication. Unnecessary tests and procedures in the health care system. 2014. Available from: w w w . c hoos in g w i s e ly. or g/w p content/uploads/2015/04/Final-ChoosingWisely-Survey-Report.pdf

10. US Congress Office of Technology Assessment. Defensive medicine and medical malpractice. Publication OTA-H602. Washington, DC: US Government Printing Office; 1994.

11. Catino M. Why do doctors practice defensive medicine? The side effects of medical litigation. Safety Sci Monit 2011;15:1-12.

12. Asher E, Parag Y, Zeller L, et al. Unconscious defensive medicine: the case of erythrocyte sedimentation rate. Eur $\mathrm{J}$ Intern Med 2007;18:35-8.
13. Summerton N. Positive and negative factors in defensive medicine: a questionnaire study of general practitioners. Brit Med J 1995;310:27-9.

14. PriceWaterhouseCoopers. The price of excess: identifying waste in healthcare spending. Arlington, VA: Pricewaterhouse Coopers' Health Research Institute; 2010.

15. Studdert D, Mello M, Sage W, et al. Defensive medicine among high-risk specialist physicians in a volatile malpractice environment. J Am Med Assoc 2005;293: 2609-17.

16. Glassman PA, Rolph JE, Petersen LP, et al. Physicians' personal malpractice experiences are not related to defensive clinical practices. J Health Polit Polic 1996;21:21941.

17. Gonzalez Morganti K, Bauhoff $S$, Blanchard JC, et al. The evolving role of emergency departments in the United States. Santa Monica, CA: RAND; 2013.

18. Sage WM. Understanding the first malpractice crisis of the 21st century. In: Gosfield AG, ed. Health law handbook. St Paul, Minn: West Group; 2003. pp 1-32.

19. Jennwood C, Majercik S, Bledsoe J, et al. The prevalence and impact of defensive medicine in the radiographic workup of the trauma patient: a pilot study. Am J Surg 2015;210:462-7.

20. Foy AJ, Liu G, Davidson WR Jr, et al. Comparative effectiveness of diagnostic testing strategies in emergency department patients with chest pain an analysis of downstream testing, interventions, and outcomes. J Am Med Assoc 2015;175:42836.

21. US Congress Office of Technology Assessment. Defensive medicine and medical malpractice. Washington, DC: US Government Printing Office; 1994.

22. La Puma J, Schiedermayer DL, Toulin S, et al. The standard of care: a case report and ethical analysis. Ann Intern Med 1988;108: 121-4.

23. Chen XY. Defensive medicine or economi- cally motivated corruption? A Confucian reflection on physician care in China today. J Med Philos 2007,32:635-48.

24. Emanuel E, Tanden N, Altman S, et al. A systemic approach to containing health care spending. New Engl J Med 2012;367: 949-54.

25. Waxman DA, Greenberg MD, Ridgely MS, et al. The effect of malpractice reform on emergency department care. New Engl J Med 2014;371:1518-25.

26. Kroenke K. Diagnostic testing and the illusory reassurance of normal results: comment on "Reassurance after diagnostic testing with a low pretest probability of serious disease." J Am Med Assoc 2013; 173:416-7.

27. Cervellin G, Borghi L, Lippi G. Do clinicians decide relying primarily on Bayesians principles or on Gestalt perception? Some pearls and pitfalls of Gestalt perception in medicine. Intern Emerg Med 2014;9:513-9.

28. Wells PS, Anderson DR, Rodger M, et al. Evaluation of D-dimer in the diagnosis of suspected deep-vein thrombosis. New Engl J Med 2003;349:1227-35.

29. Casagranda I, Cavazza M, Clerico A, et al. Proposal for the use in emergency departments of cardiac troponins measured with the latest generation methods in patients with suspected acute coronary syndrome without persistent ST-segment elevation. Clin Chem Lab Med 51:1727-37.

30. US Institute of Medicine. Committee on the health professions education summit. In: Greiner AC, Knebel E, eds. Health professions education: a bridge to quality. Washington, DC: National Academies Press; 2003. pp 153-55.

31. O'Leary KJ, Choi J, Watson K, Williams MV. Medical students' and residents' clinical and educational experiences with defensive medicine. Acad Med 2012;87:142-8. 